\title{
Myofibroblast distribution is associated with invasive growth types of colorectal cancer
}

\author{
MASAFUMI TAKATSUNA ${ }^{1,2}$, SATOKO MOROHASHI ${ }^{1}$, TADASHI YOSHIZAWA ${ }^{1}$, HIDEAKI HIRAI ${ }^{1}$, \\ TOSHIHIRO HAGA ${ }^{1}$, RIE OTA ${ }^{1}$, KENSUKE SAITO ${ }^{1}$, YUNYAN WU ${ }^{1}$, HIROKO SEINO ${ }^{1}$, \\ YUTAKA AOYAGI $^{3}$, SHUJI TERAI ${ }^{2}$ and HIROSHI KIJIMA ${ }^{1}$ \\ ${ }^{1}$ Department of Pathology and Bioscience, Hirosaki University Graduate School of Medicine, Hirosaki 036-8562; \\ ${ }^{2}$ Division of Gastroenterology and Hepatology, Graduate School of Medical and Dental Sciences, Niigata University, \\ Chuo-ku, Niigata 951-8510; ${ }^{3}$ Department of Gastroenterology, Niigata Medical Center, \\ Niigata Prefectural Federation of Agricultural Cooperatives for Health and Welfare, \\ Nishi-ku, Niigata 950-2022, Japan
}

Received February 26, 2016; Accepted April 7, 2016

DOI: $10.3892 /$ or.2016.5202

\begin{abstract}
Both the invasive growth types of colorectal cancer (CRC) and the number of myofibroblasts have been associated with histopathological factors such as lymph node and liver metastasis, and local recurrence. However, there are few studies, that have assessed the association between invasive growth type and myofibroblast distribution in CRC. We aimed to evaluate the relationship between the clinicopathological factors of CRC and two invasive growth types, the expanding and infiltrating types. We categorized 150 cases of pT3 CRC into the expanding and infiltrating types and measured the myofibroblast density of three histological layers: the submucosa (SM), the muscularis propria (MP) and the subserosa (SS). We compared these two invasive growth types and analyzed the relationship between clinicopathological factors and myofibroblast density. Myofibroblast density was significantly higher in the infiltrating type than that in the expanding type $(\mathrm{P}<0.05)$. In the lymph node metastasis-positive group of the infiltrating type, myofibroblast density in MP was significantly higher than that in the lymph node metastasis-negative group $(\mathrm{P}<0.001)$. In the infiltrating type, the group with the higher level of lymphatic invasion had a significantly higher density of myofibroblasts in the MP than the group with the lower level of lymphatic invasion $(\mathrm{P}<0.01)$. These results suggest that myofibroblasts participate more in the infiltrating type
\end{abstract}

Correspondence to: Dr Satoko Morohashi, Department of Pathology and Bioscience, Hirosaki University Graduate School of Medicine, 5 Zaifu-cho, Hirosaki 036-8562, Japan

E-mail: msatoko@hirosaki-u.ac.jp

\section{Abbreviations: CRC, colorectal cancer}

Key words: colorectal cancer, myofibroblast, invasive growth type, lymphatic invasion, lymph node metastasis compared with the expanding type of CRC. It would appear that myofibroblasts present in the MP play an important role in the malignant potential of the infiltrating type compared to the expanding type.

\section{Introduction}

According to the World Health Organization, colorectal cancer (CRC) is one of the most lethal diseases worldwide (1). The tumor-node-metastasis (TMN) classification is based on the standard staging process, which helps understand the histopathological features of CRC and is an important factor in deciding its prognosis. The invasive growth types have also been evaluated as valuable prognostic factors $(2,3)$. In patients with CRC, the invasive growth type influences local recurrence, liver metastasis and disease-free survival (2-5).

In contrast, the association between CRC and myofibroblasts in the tumor microenvironment has only recently attracted considerable attention. Although the myofibroblast is known as a principal cellular component in the granulation tissue of healing wounds, cancer stromal cells containing myofibroblasts construct the extracellular matrix (ECM) $(6,7)$. The ECM not only plays a role in one of the scaffolding structures but also influences cancer proliferation, and activities of invasion and metastasis $(6,8)$. In patients with CRC, there is a correlation between the concentration of tumor stroma and patient cumulative survival (9). The myofibroblasts in the stroma of CRC also serve an important function in promoting the desmoplastic stromal reaction and influence tumor invasion, microvessel density around the invasive lesion and metastatic carcinomas (10-12). Moreover, myofibroblast activation in tumor metastatic lymph nodes influences the microenvironment supporting CRC metastasis (13).

In both the invasion and metastasis of CRC, three histological layers of the colorectum, the submucosa (SM), the muscularis propria (MP) and the subserosa (SS), may play an important role in the mechanical and physiological protection against the invasive growth of CRC. MP is composed 
exclusively of smooth muscle bundles and comprises tight connective tissue, whereas SM and SS are composed mainly of loose connective tissue $(14,15)$. However, it is unclear how myofibroblasts are distributed around the CRC invasive border of these three layers and whether there is an association between the invasive growth type of CRC and the distribution of myofibroblasts around the invasive lesions.

In the present study, we focused on the association between the invasive growth type of CRC and myofibroblast distribution in SM, MP and SS layers, separately. Furthermore, we investigated the association between the density of myofibroblasts and the clinicopathological factors such as lymph node metastasis and venous invasion, supported by immunohistochemical staining and imaging analysis.

\section{Materials and methods}

Patients and tissues. One hundred and fifty patients with advanced invasive CRC, defined as adenocarcinoma, which had invaded the subserosal layer of the colorectal wall (pT3), underwent surgical resection from January 2008 to December 2009 at Hirosaki University Hospital. All patients were not treated with neoadjuvant chemotherapy and did not have synchronous multiple CRCs. We used surgically resected specimens that were fixed with $10 \%$ formalin, then embedded in paraffin and stained with hematoxylin and eosin $(H \& E)$ for pathological evaluation. We confirmed whether or not they had liver metastasis by referring to medical records from when the 150 patients underwent operative resection.

Pathological analysis. We divided the 150 cases into two invasive growth types. For the purposes of the present study, we described these invasive growth types as the expanding and infiltrating types. The expanding and infiltrating types are shown in Fig. 1A and B. A representative case of the expanding (Fig. 1C) and infiltrating type (Fig. 1D) with adenocarcinoma lesion of the MP (H\&E staining) are shown. The expanding type was defined as the overall expansion growth type of adenocarcinoma, with a clear invasive margin. The infiltrating type was defined as a widespread streaming form of adenocarcinoma. These two novel defined types are different from the INF classification since the INF classification specifically denotes lesions with borderline tumor invasive properties (2). Degrees of lymphatic and venous vessel invasion were classified as 0 , no invasion; 1 , mild invasion; 2 , moderate invasion and 3 , severe invasion. Several histological features were assessed: the location of the primary adenocarcinoma, the dominant histological type and lymph node metastasis. These parameters referred to the Japanese Classification of Colorectal Carcinoma (16).

Immunohistochemistry. We selected the paraffin-embedded specimen which recognized three colorectal wall layers (SM, MP and SS), and the specimen also had the invasive lesion of the adenocarcninoma diagnosed by H\&E staining. The selected paraffin embedded specimen was a representative block of each case, and we used $4-\mu \mathrm{m}$ serial sections for immunohistochemistry. The sections were mounted on saline-coated glass slides. The antibodies used included $\alpha$-smooth muscle actin ( $\alpha$-SMA) (1:100; clone 1A4) and desmin
(1:100; clone D-33) (both from Dako, Glostrup, Denmark). Immunostaining for $\alpha$-SMA and desmin was performed using the standard avidin-biotin-peroxidase complex method with an automated immunostainer (BenchMark XT; Ventana Medical System, Tucson, AZ, USA). The signature characteristic of the myofibroblast was the $\alpha$-SMA-positive and desmin-negative pattern, whereas that of smooth muscle was the $\alpha$-SMA- and desmin-positive pattern.

Imaging analysis. We used imaging analysis to investigate myofibroblast density. Each invasive growth type had an invasive lesion of the three colorectal walls, SM, MP and SS. To obtain all images, we used an Olympus microscope BX50 with U PlanApo objective lens (magnification, $x 4$ ), DP control software and a digital camera DP-70 (all from Olympus, Tokyo, Japan). We applied ImageJ software (NIH, Bethesda, MD, USA) to view and analyze our obtained images (17). The images of both $\alpha$-SMA and desmin staining were binarized. We conducted a subtraction image pasting the binarized image of desmin onto the binarized images of $\alpha$-SMA using the subtraction mode in ImageJ software. The subtraction images were shown as the value of $\alpha$-SMA minus that of desmin and we could interpret the subtraction image as the existence of myofibroblasts in the representative section of each case. The representative images of the expanding and infiltrating types which invaded the MP layer are shown in Fig. 2A-H, respectively. From all 150 cases, we obtained the subtraction image of the three histological layers (SM, MP and SS) and measured the myofibroblast density of the $1 \times 1 \mathrm{~mm}^{2}$ area in the invasive border of each layer. We selected a hotspot myofibroblast density area from each invasive lesion (Fig. 2D and H).

Statistical analysis. All values are presented as the means \pm standard error of the mean (SEM). A Chi-square test for non-continuous variables was performed, whereas the Mann-Whitney and Welch t-tests were used for continuous variables in comparing each parameter. Differences were considered to indicate a statistically significant result, when the P-value was $<0.05$. Statistical analysis was performed with R (http://www.r-project.org) and Microsoft Excel software (Microsoft Corporation, Redmond, WA, USA).

\section{Results}

Difference in clinicopathological characteristics between the expanding and infiltrating types of $C R C$. We classified the growth type of $150 \mathrm{CRC}$ resections into either the expanding (67 cases) or the infiltrating type (83 cases) according to our definition (Fig. 1). The clinicopathological characteristics are summarized in Table I. With respect to age, gender, location and histological type, there was no significant difference between the expanding and infiltrating types. Twenty-seven patients $(40.3 \%)$ with the expanding type of cancer had lymph node metastasis and 55 patients $(66.3 \%)$ with the infiltrating type of cancer had lymph node metastasis. The percentage of low or high lymphatic invasion with the expanding type was 85.1 and $14.9 \%$, respectively. In contrast, the percentage of low and high lymphatic invasion which existed in the infiltrating type was 42.1 and $57.9 \%$, respectively. The percentage of low and high venous invasion with the expanding type was 80.6 

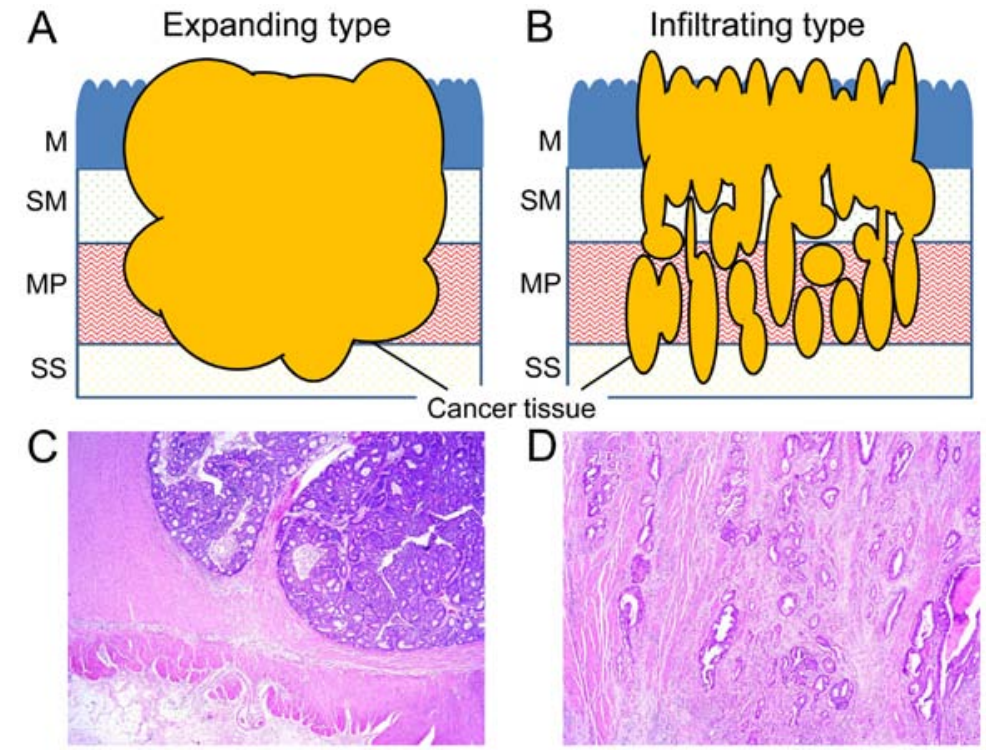

Figure 1. Expanding and infiltrating types of colorectal cancer, which invade through the mucosa to the subserosa. (A) The expanding type was recognized as the overall expansion growth type of adenocarcinoma and the invasive margin was clear. (B) The infiltrating type was recognized as the widespread streaming form of adenocarcinoma. Histology of the (C) expanding and the (D) infiltrating types around the tumor invasive lesion in the muscularis propria layer. M, mucosa; SM, submucosa; MP, muscularis propria; SS, subserosa.

\section{Expanding type}

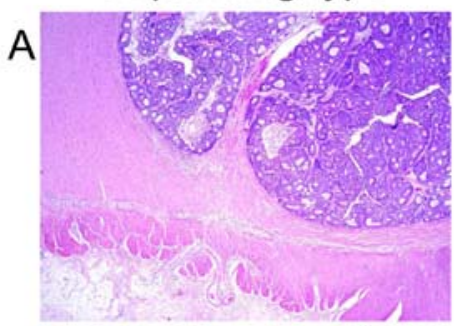

B
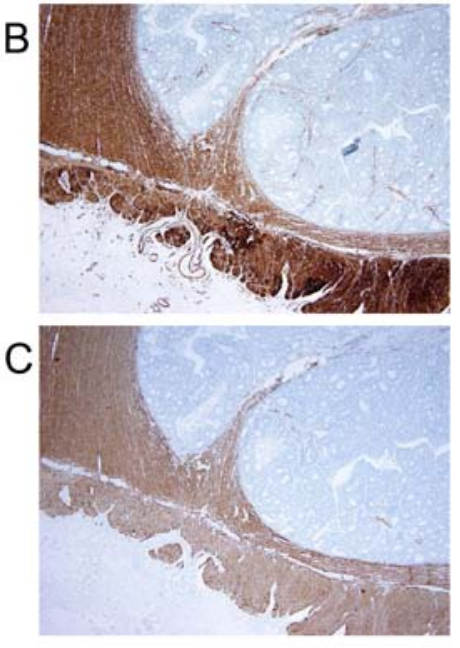

D

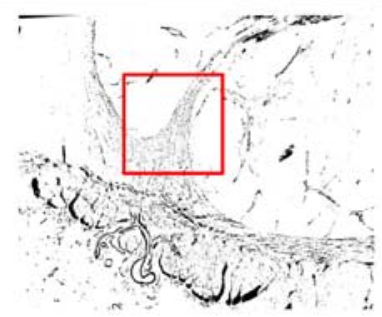

Infiltrating type
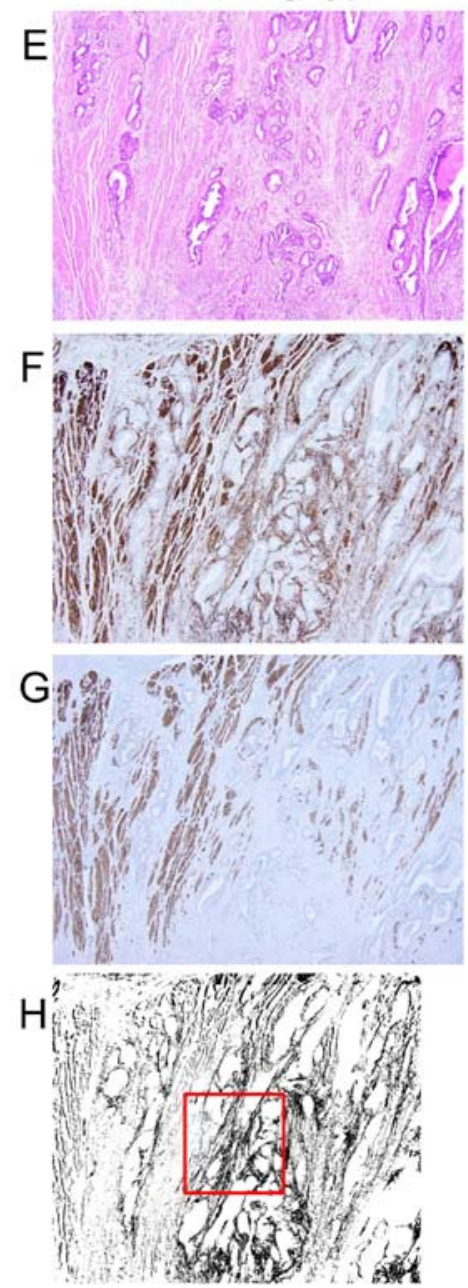

Figure 2. Representations of the expanding type around the invasive lesion of the muscularis propria layer: (A) hematoxylin and eosin staining, (B) $\alpha$-SMA, (C) desmin and (D) subtraction image ( $\alpha$-SMA-desmin). Representations of the infiltrating type around the invasive lesion of the muscularis propria layer: (E) hematoxylin and eosin staining, (F) $\alpha$-SMA, (G) desmin and (H) subtraction image ( $\alpha$-SMA-desmin). The red square is a $1 \times 1 \mathrm{~mm}^{2}$ area, which measures the myofibroblast density (D and $\mathrm{H})$. 
Table I. Differences in the clinicopathological characteristics between the expanding and the infiltrating types.

\begin{tabular}{|c|c|c|c|}
\hline Variables & $\begin{array}{c}\text { Expanding } \\
\mathrm{n}=67\end{array}$ & $\begin{array}{c}\text { Infiltrating } \\
n=83\end{array}$ & P-value \\
\hline $\begin{array}{l}\text { Age, years } \\
\text { median (range) }\end{array}$ & $65.6(26-87)$ & $66.6(39-93)$ & 0.655 \\
\hline Gender, n & & & 0.314 \\
\hline Male & 41 & 44 & \\
\hline Female & 26 & 39 & \\
\hline Location, $\mathrm{n}$ & & & 0.353 \\
\hline Cecum & 4 & 2 & \\
\hline Ascending & 13 & 14 & \\
\hline Transverse & 10 & 4 & \\
\hline Descending & 3 & 4 & \\
\hline Sigmoid & 14 & 21 & \\
\hline Rectal & 23 & 38 & \\
\hline Histological type, $\mathrm{n}$ & & & 0.105 \\
\hline Well and mod & 60 & 79 & \\
\hline Por & 4 & 3 & \\
\hline Muc & 3 & 1 & \\
\hline \multicolumn{3}{|c|}{ Lymph node metastasis, n (\%) } & 0.001 \\
\hline $\mathrm{pN}(-)$ & $40(59.7)$ & $28(33.7)$ & \\
\hline $\mathrm{pN}(+)$ & $27(40.3)$ & $55(66.3)$ & \\
\hline \multicolumn{3}{|l|}{ Lymphatic invasion, $\mathrm{n}(\%)$} & $<0.0001$ \\
\hline Low (ly0 or ly 1$)$ & $57(85.1)$ & $35(42.1)$ & \\
\hline High (ly 2 or ly 3 ) & $10(14.9)$ & $48(57.9)$ & \\
\hline Venous invasion, $\mathrm{n}(\%)$ & & & 0.003 \\
\hline Low (v0 or v1) & $54(80.6)$ & $52(62.7)$ & \\
\hline High (v2 or v3) & $13(19.4)$ & $31(37.3)$ & \\
\hline Liver metastasis $(+), \mathrm{n}$ & 3 & 6 & \\
\hline
\end{tabular}

Well, well-differentiated adenocarcinoma; mod, moderately differentiated adenocarcinoma; Por, poorly differentiated adenocarcinoma; Muc, mucinous adenocarcinoma; ly, lymphatic invasion; v, venous invasion.

and $19.4 \%$, respectively, while the percentage of low and high venous invasion which existed in the infiltrating type was 62.7 and $37.3 \%$, respectively. Three patients with the expanding type had liver metastasis, while 6 patients with the infiltrating type had liver metastasis. With respect to lymph node metastasis, lymphatic invasion and venous invasion, there were significant differences between the expanding and infiltrating types $(\mathrm{P}<0.003)$.

Myofibroblast distribution in the invasive lesion at each colorectal wall stratified by expanding type vs. infiltrating type. We measured the myofibroblast density around the invasive front of each layer (SM, MP and SS) for the expanding and infiltrating types (Fig. 3). In 67 cases of the expanding type, the mean myofibroblast density for each layer of the invasive legion was $11.03 \pm 0.88 \%(\mathrm{SM}), 11.62 \pm 0.50 \%(\mathrm{MP})$ and $19.24 \pm 1.34 \%$ (SS). In contrast, in 83 cases of the infiltrating

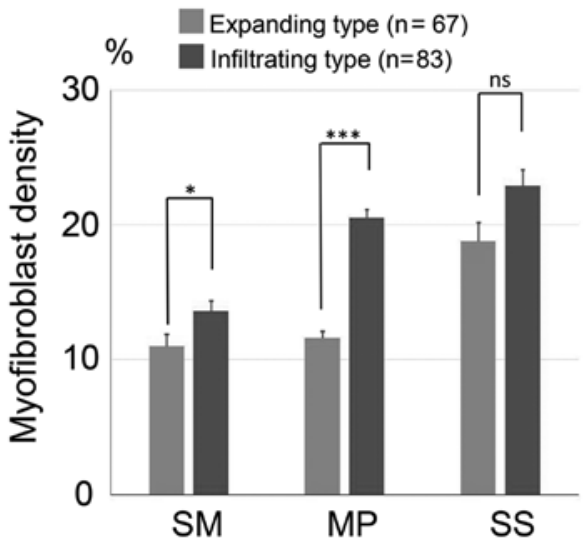

Figure 3. Mean myofibroblast density in each layer around the invasive lesion of colorectal cancer. In both the SM and the MP, there was a significant difference in myofibroblast density between the expanding and infiltrating types. Values are given as the mean \pm SEM. ${ }^{*} \mathrm{P}<0.05,{ }^{* * *} \mathrm{P}<0.001$ for the expanding vs. the infiltrating type. ns, not significant; SM, submucosa; MP, muscularis propria; SS, subserosa.

type, the mean myofibroblast density was $13.60 \pm 0.79 \%$ (SM), $20.52 \pm 0.62 \%$ (MP) and $22.40 \pm 1.07 \%$ (SS). Significantly more myofibroblasts were located around the invasive lesion of the SM $(\mathrm{P}=0.018)$ and the MP $(\mathrm{P}<0.001)$ in the infiltrating type than in the expanding type.

Association between the myofibroblast distribution, the invasive growth types and lymph node metastasis. We stratified 67 cases of the expanding type and 83 cases of the infiltrating type into lymph node metastasis-negative and a -positive groups. Then, we investigated myofibroblast distribution of the three invasive layers (Fig. 4A and B). In the expanding type, the mean myofibroblast densities of the three layers within the lymph node metastasis-negative group $(\mathrm{n}=40)$ were $11.58 \pm 1.31 \%$ (SM), $11.64 \pm 0.69 \%$ (MP) and $19.35 \pm 1.56 \%$ (SS), while the mean myofibroblast densities in the lymph node metastasis-positive group $(\mathrm{n}=27)$ were $10.25 \pm 1.04 \%$ (SM), $11.59 \pm 0.71 \%$ (MP) and $19.06 \pm 2.42 \%$ (SS). In the expanding type, there was no significant difference in myofibroblast densities of each colorectal wall layer between the lymph node metastasis-positive and the -negative groups. In the infiltrating type, the mean myofibroblast densities of the three layers within the lymph node-negative group $(\mathrm{n}=28)$ were $12.93 \pm 1.33 \%$ (SM), $17.55 \pm 0.66 \%$ (MP) and $20.54 \pm 1.56 \%$ (SS), while the mean myofibroblast densities in the lymph node-positive group $(\mathrm{n}=55)$ were $13.94 \pm 0.99 \%$ (SM), $22.04 \pm 0.80 \%$ (MP) and $23.34 \pm 1.38 \%$ (SS). In the infiltrating type, the lymph node-positive group had a higher myofibroblast density for each layer than the lymph node-negative group. Furthermore there was a significant difference between the lymph node metastasis-positive and metastasis-negative groups relating to the myofibroblast density of the MP layer $(\mathrm{P}<0.001)$.

Association between the distribution of myofibroblast density, invasive growth types and lymphatic vessel invasion. To investigate the association between the distribution of myofibroblasts and the degree of lymphatic vessel invasion, we stratified the 67 cases of the expanding type and 83 cases of the infiltrating type into either low lymphatic vessel invasion 

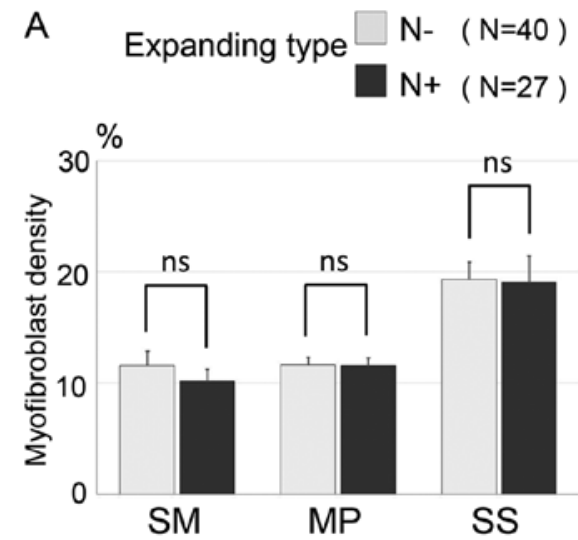

B Infiltrating type $\square \mathrm{N}-(\mathrm{N}=28)$

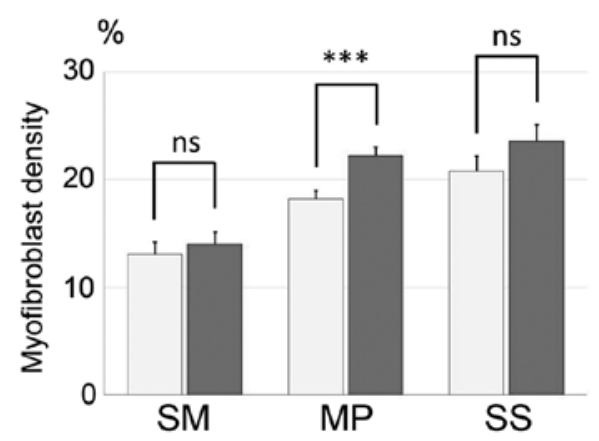

Figure 4. Mean myofibroblast density of lymph node metastasis-negative and -positive groups in the (A) expanding and the (B) infiltrating types. There was a significant difference between the lymph node metastasis-positive and metastasis-negative groups in regards to the myofibroblast density in the MP colorectal wall in the infiltrating type. Values are given as the mean $\pm \mathrm{SEM}$. ${ }^{* * *} \mathrm{P}<0.001$ the lymph node-negative group vs. the lymph node-positive group in regards to the infiltrating type. ns, not significant; SM, submucosa; MP, muscularis propria; SS, subserosa.
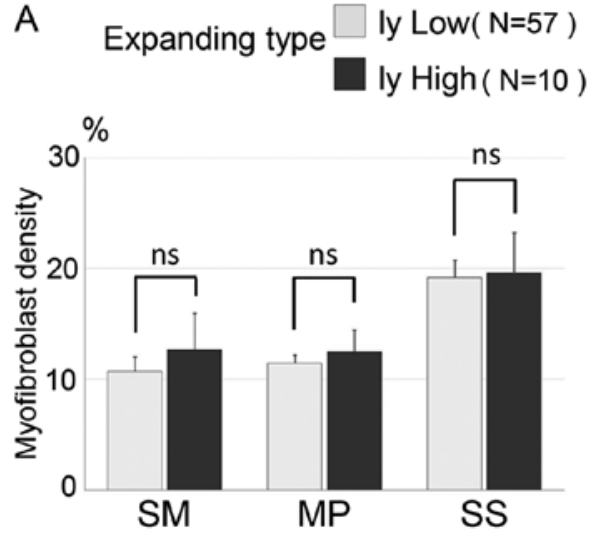

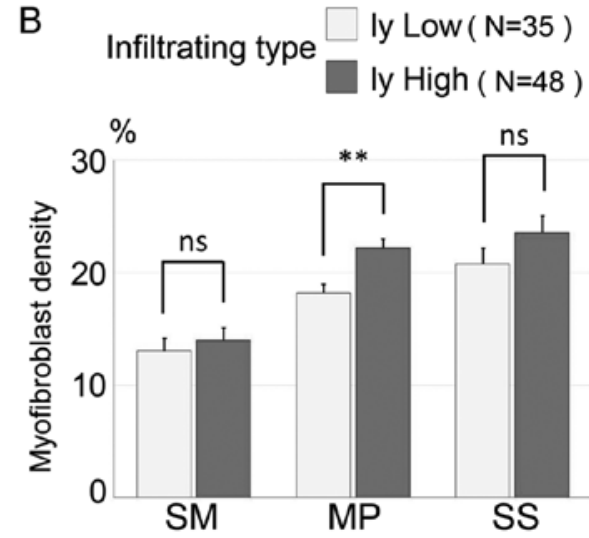

Figure 5. Mean myofibroblast density in the low lymphatic vessel invasion and high lymphatic vessel invasion groups in the (A) expanding and the (B) infiltrating types. In the infiltrating type, there was a significant difference between the low and high lymphatic vessel invasion groups in regards to the myofibroblast density in the MP colorectal wall. Values are given as the mean $\pm \mathrm{SEM} .{ }^{* *} \mathrm{P}<0.01$ the low lymphatic vessel invasion group vs. the high lymphatic vessel invasion group in regards to the infiltrating type. ns, not significant; SM, submucosa; MP, muscularis propria; SS, subserosa.

or high lymphatic vessel invasion groups. We analyzed the distribution of myofibroblasts around the three colorectal wall layers (Fig. 5A and B). In the expanding type, the mean myofibroblast densities in each of the three layers within the low lymphatic vessel invasion group $(n=57)$ were $10.74 \pm 0.87 \%$ (SM), $11.47 \pm 0.48 \%$ (MP) and $19.18 \pm 1.45 \%(\mathrm{SS})$, and the mean myofibroblast densities in the high lymphatic vessel invasion group $(n=10)$ were $12.66 \pm 3.30 \%$ (SM), $12.48 \pm 1.98 \%$ (MP) and $19.58 \pm 3.64 \%$ (SS). There was no significant difference between the low and high groups in the expanding type. In the infiltrating type, the mean myofibroblast densities for each of the three layers in the low lymphatic vessel invasion group $(n=35)$ were $13.07 \pm 1.10 \%(\mathrm{SM}), 18.27 \pm 0.80 \%$ (MP) and $20.79 \pm 1.39 \%(\mathrm{SS})$, and the mean myofibroblast densities in the high lymphatic vessel invasion group $(n=48)$ were $13.39 \pm 1.11 \%$ (SM), $22.22 \pm 0.80 \%$ (MP) and $23.57 \pm 1.52 \%(\mathrm{SS})$. In the infiltrating type, there was a significant difference in myofibroblast density of the MP layer between the low and high lymphatic vessel invasion group $(\mathrm{P}=0.007)$.

Association between the distribution of myofibroblast density, invasive growth types and venous vessel invasion.
To investigate the association between the distribution of myofibroblasts and the degree of venous vessel invasion, we stratified the 67 cases of the expanding type and the 83 cases of the infiltrating type into a low venous vessel invasion and a high venous vessel invasion group and analyzed the distribution of myofibroblasts around each of the three colorectal walls (Fig. 6A and B). In the expanding type, the mean myofibroblast densities of each layer in the low venous invasion group $(n=54)$ were $10.72 \pm 0.92 \%(\mathrm{SM}), 11.37 \pm 0.48 \%$ (MP) and $18.69 \pm 1.48 \%$ (SS), while the mean myofibroblast densities in the high venous vessel invasion group $(\mathrm{n}=13)$ were $12.31 \pm 2.49 \%(\mathrm{SM}), 12.65 \pm 1.69 \%(\mathrm{MP})$ and $21.51 \pm 3.15 \%(\mathrm{SS})$. There was no significant difference between the low venous vessel and high venous vessel invasion groups within the expanding type. In the infiltrating type, the mean myofibroblast densities of each layers in the low venous invasion group ( $\mathrm{n}=52$ ) were $13.77 \pm 0.92 \%$ (SM), $20.85 \pm 0.78 \%(\mathrm{MP})$ and $22.95 \pm 1.27 \%$ (SS) and the mean myofibroblast densities in the high venous vessel invasion group $(n=31)$ were $13.77 \pm 1.26 \%$ (SM), $19.98 \pm 1.02 \%$ (MP) and $21.47 \pm 1.90 \%$ (SS). There was no significant difference between the low and high venous vessel invasion groups within the infiltrating type. 
A
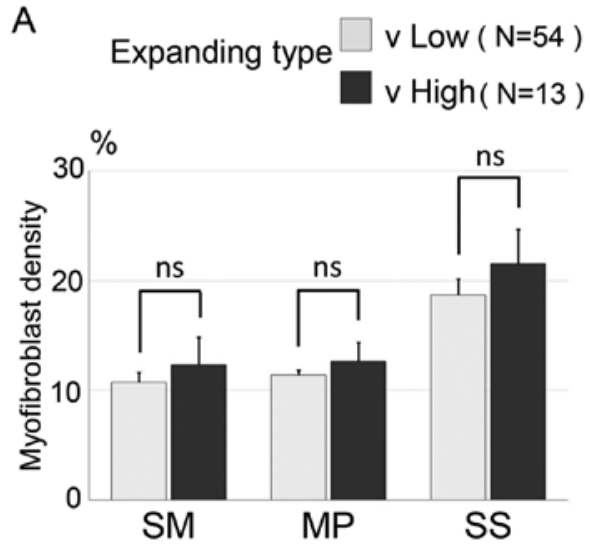

B
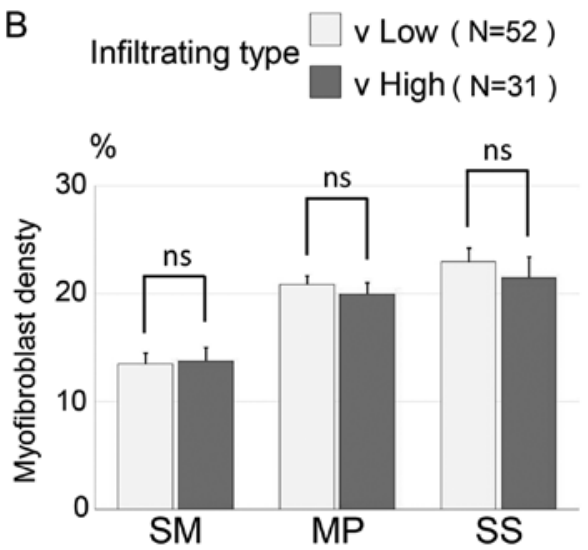

Figure 6. Mean myofibroblast density in the low venous invasion and high venous invasion groups in the (A) expanding and (B) infiltrating types. Values are given as the mean \pm SEM. ns, not significant; SM, submucosa; MP, muscularis propria; SS, subserosa.

\section{Discussion}

In the present study, we found that the infiltrating type of colorectal cancer (CRC) was aggressive, as evidenced by lymph node metastasis and by lymphatic and venous invasion, and was more progressive than the expanding type. Previous studies identified that the invasive growth type correlates strongly with liver metastasis and is one of the most important factors that determines prognosis for patients with CRC $(3,4,18,19)$. Moreover, the infiltrating type carries a high risk of liver metastasis and a worse prognosis compared to the expanding type $(4,20)$. Although the definition of growth type is different between our research and previous studies, we suspect that the present study reflects prior investigations.

The present study revealed that the quantity of myofibroblasts which were located around the CRC invasive lesion was significantly different between the infiltrating and expanding types. Myofibroblasts are considered to be a type of cancer-associated fibroblasts $(\mathrm{CAFs})$ and to be involved in the desmoplastic reaction (21). CAFs actively associate with neoplastic cells and form a myofibroblastic microenvironment that promotes cancer growth, angiogenesis and survival, which supports malignancy (22). CAFs are not present peritumorally as individual cells, but they combine to fully deploy a desmoplastic program which is associated with CRC malignancy, and they have an important role in the prognosis of CRC patients (21-23). Therefore, we suggest that the infiltrating type induces a high number of myofibroblasts which give rise to a very strong desmoplastic reaction via interaction of CAFs with malignant potential.

We found that as the invasion of the CRC became deeper, the number of myofibroblasts increased around the invasive lesions, and the invasive growth type of CRC had a significantly higher density of myofibroblasts than the expanding type. It is possible that the large quantity of myofibroblasts altered the adhesive and migratory properties of CRC cells, which consequently aided CRC invasion into the deeper layers of the SM, MP and SS. Myofibroblasts promote CRC invasion and metastasis as they proliferate around the invasive lesion and alter the adhesive and migratory properties of CRC cells $(12,24)$. The relationship between the adhesion molecule (for example E-cadherin) and the malignant potential of CRC is debated $(25,26)$. However, one study showed that myofibroblasts co-cultured with CRC cells may be involved in the invasiveness of CRC, even when E-cadherin expression prevents tumor cell invasiveness in vitro (27). In contrast, in previous studies, myofibroblasts at the deep border of CRC may reduce the invasive activity of CRC cells, and entrap tumor cells since myofibroblasts have been shown to be involved in the wound repair process, and desmoplastic reactions are known to be involved in local remodeling in the healing of wounds $(14,28)$. However, such myofibroblasts are thought to arise from resident fibroblasts, which are transiently present (14). We suggest that this type of myofibroblast is different from the myofibroblasts located near the CRC invasive lesion. Therefore, the present study predicts that myofibroblasts located next to the invasive lesion accelerate invasive growth by altering the adhesion and migratory system of CRC, comparable to a cellular foothold. Moreover, the infiltrating type may be particularly relevant relating to this effect compared to the expanding type.

Our results showed that the myofibroblast density of MP was high in both the lymph node metastasis-positive and the high lymphatic vessel invasion groups, particularly in the infiltrating type. The lymphatic vessels exist in three colorectal wall levels, the SM, MP and SS despite the differences regarding histological structure. The distribution of lymphatic vessels in normal colonic tissue tends to increase in frequency with depth throughout the wall (29). We suggest that myofibroblasts invade through the connective tissue of the MP and are strongly associated with lymphatic invasion of CRC in the MP compared with the SM and SS, which have loose connective tissue. The functions of $\alpha$-SMA-positive myofibroblasts may be associated with the promotion of the ECM of tumor cells and lymphogenesis of the metastatic microenvironment in oral tongue squamous cell carcinoma (30). With respect to CRC, proliferation of myofibroblasts in the peritumoral areas was predicted to play an important role in lymphangiogenesis, and was also associated with lymph node metastasis (12). Moreover, the finding that CRC-invading MP may result in a greater ability to induce angiogenesis in adjacent normal tissue has been reported by other studies (31). The present study predicted that myofibroblasts participate in both lymphangiogenesis and lymphatic vessel invasion of the MP layer, 
which affects lymph node metastasis of the infiltrating type compared with the expanding type.

In conclusion, we revealed that the infiltrating type of $\mathrm{CRC}$ has a greater malignant potential than the expanding type through myofibroblast contribution. Furthermore, we have shown that myofibroblasts present in the MP play a more important role in the malignant potential of the infiltrating type than that of the expanding type.

\section{Acknowledgements}

The present study was supported by Grants-in-Aid for Science from the Ministry of Education, Culture, Sports, Science and Technology in Japan, and a grant for Hirosaki University Institutional Research.

\section{References}

1. Bosman FT, Carneiro F, Hruban RH and Theise ND (eds): World Health Organization Classification of Tumours of the Digestive system. Vol 3. 4th edition. IARC, Lyon, pp132-146, 2010.

2. Hase H, Mochizuki H, Utsunomiya K, Iwamoto K, Kuranaga K, Watanabe C and Tamakuma S: A study on prognostic value of infiltrative growth (INF) in patients with colorectal cancer. J Jpn Soc Coloproctol 49: 463-467, 1996.

3. Pinheiro RS, Herman P, Lupinacci RM, Lai Q, Mello ES, Coelho FF, Perini MV, Pugliese V, Andraus W, Cecconello I, et al: Tumor growth pattern as predictor of colorectal liver metastasis recurrence. Am J Surg 207: 493-498, 2014.

4. Rajaganeshan R, Prasad R, Guillou PJ, Chalmers CR, Scott N, Sarkar R, Poston G and Jayne DG: The influence of invasive growth pattern and microvessel density on prognosis in colorectal cancer and colorectal liver metastases. Br J Cancer 96: 1112-1117, 2007.

5. Fujita S, Shimoda T, Yoshimura K, Yamamoto S, Akasu T and Moriya Y: Prospective evaluation of prognostic factors in patients with colorectal cancer undergoing curative resection. J Surg Oncol 84: 127-131, 2003.

6. Bissell MJ and Radisky D: Putting tumours in context. Nat Rev Cancer 1: 46-54, 2001.

7. Seemayer TA, Schürch W and Lagacé R: Myofibroblasts in human pathology. Hum Pathol 12: 491-492, 1981.

8. Kalluri R and Weinberg RA: The basics of epithelial-mesenchymal transition. J Clin Invest 119: 1420-1428, 2009.

9. Park JH, Richards CH, McMillan DC, Horgan PG and Roxburgh CS: The relationship between tumour stroma percentage, the tumour microenvironment and survival in patients with primary operable colorectal cancer. Ann Oncol 25: 644-651, 2014.

10. Okamoto Y, Fujimori T, Ohkura Y, Sugai T, Arai T, Watanabe G, Wada R, Ueno H, Togashi K, Yao T, et al: Histological assessment of intra- and inter-institutional reliabilities in detection of desmoplastic reaction in biopsy specimens of early colorectal carcinomas. Pathol Int 63: 539-545, 2013.

11. Tsujino T, Seshimo I, Yamamoto H, Ngan CY, Ezumi K, Takemasa I, Ikeda M, Sekimoto M, Matsuura N and Monden M: Stromal myofibroblasts predict disease recurrence for colorectal cancer. Clin Cancer Res 13: 2082-2090, 2007.

12. Liang P, Hong JW, Ubukata H, Liu G, Katano M, Motohashi G, Kasuga T, Watanabe Y, Nakada I and Tabuchi T: Myofibroblasts correlate with lymphatic microvessel density and lymph node metastasis in early-stage invasive colorectal carcinoma. Anticancer Res 25: 2705-2712, 2005.

13. Yeung TM, Buskens C, Wang LM, Mortensen NJ and Bodmer WF: Myofibroblast activation in colorectal cancer lymph node metastases. Br J Cancer 108: 2106-2115, 2013.
14. Nakayama H, Enzan H, Miyazaki E, Naruse K, Kiyoku H and Hiroi M: The role of myofibroblasts at the tumor border of invasive colorectal adenocarcinomas. Jpn J Clin Oncol 28: 615-620, 1998.

15. Ueno H, Hase K, Hashiguchi Y, Ishiguro M, Kajiwara Y, Shimazaki $\mathrm{H}$ and Mochizuki H: Growth pattern in the muscular layer reflects the biological behaviour of colorectal cancer. Colorectal Dis 11: 951-959, 2009.

16. Japanese Society for Cancer of the Colon and Rectum: Japanese Classification of Colorectal Carcinoma. 2nd English edition. Kanehara \& Co., Ltd, Tokyo, 2009.

17. Schneider CA, Rasband WS and Eliceiri KW: NIH Image to ImageJ: 25 years of image analysis. Nat Methods 9: 671-675, 2012.

18. Jass JR, Love SB and Northover JM: A new prognostic classification of rectal cancer. Lancet 1: 1303-1306, 1987.

19. Jass JR, Ajioka Y, Allen JP, Chan YF, Cohen RJ, Nixon JM, Radojkovic M, Restall AP, Stables SR and Zwi LJ: Assessment of invasive growth pattern and lymphocytic infiltration in colorectal cancer. Histopathology 28: 543-548, 1996.

20. Morikawa T, Kuchiba A, Qian ZR, Mino-Kenudson M, Hornick JL, Yamauchi M, Imamura Y, Liao X, Nishihara R, Meyerhardt JA, et al: Prognostic significance and molecular associations of tumor growth pattern in colorectal cancer. Ann Surg Oncol 19: 1944-1953, 2012.

21. Karagiannis GS, Petraki C, Prassas I, Saraon P, Musrap N, Dimitromanolakis A and Diamandis EP: Proteomic signatures of the desmoplastic invasion front reveal collagen type XII as a marker of myofibroblastic differentiation during colorectal cancer metastasis. Oncotarget 3: 267-285, 2012.

22. Karagiannis GS, Poutahidis T, Erdman SE, Kirsch R, Riddell RH and Diamandis EP: Cancer-associated fibroblasts drive the progression of metastasis through both paracrine and mechanical pressure on cancer tissue. Mol Cancer Res 10: 1403-1418, 2012.

23. Ueno H, Shinto E, Shimazaki H, Kajiwara Y, Sueyama T, Yamamoto J and Hase K: Histologic categorization of desmoplastic reaction: Its relevance to the colorectal cancer microenvironment and prognosis. Ann Surg Oncol 22: 1504-1512, 2015.

24. Martin M, Pujuguet P and Martin F: Role of stromal myofibroblasts infiltrating colon cancer in tumor invasion. Pathol Res Pract 192: 712-717, 1996.

25. Kitadai Y, Ellis LM, Tucker SL, Greene GF, Bucana CD, Cleary KR, Takahashi Y, Tahara E and Fidler IJ: Multiparametric in situ mRNA hybridization analysis to predict disease recurrence in patients with colon carcinoma. Am J Pathol 149: 1541-1551, 1996.

26. Gofuku J, Shiozaki H, Tsujinaka T, Inoue M, Tamura S, Doki Y, Matsui S, Tsukita S, Kikkawa N and Monden M: Expression of E-cadherin and alpha-catenin in patients with colorectal carcinoma. Correlation with cancer invasion and metastasis. Am J Clin Pathol 111: 29-37, 1999.

27. Dimanche-Boitrel MT, Vakaet L Jr, Pujuguet P, Chauffert B, Martin MS, Hammann A, Van Roy F, Mareel M and Martin F: In vivo and in vitro invasiveness of a rat colon-cancer cell line maintaining E-cadherin expression: An enhancing role of tumorassociated myofibroblasts. Int J Cancer 56: 512-521, 1994.

28. Hewitt RE, Powe DG, Carter GI and Turner DR: Desmoplasia and its relevance to colorectal tumour invasion. Int J Cancer 53: 62-69, 1993.

29. Duff SE, Jeziorska M, Kumar S, Haboubi N, Sherlock D, O'Dwyer ST and Jayson GC: Lymphatic vessel density, microvessel density and lymphangiogenic growth factor expression in colorectal cancer. Colorectal Dis 9: 793-800, 2007.

30. Ding L, Zhang Z, Shang D, Cheng J, Yuan H, Wu Y, Song X and Jiang $H$ : $\alpha$-Smooth muscle actin-positive myofibroblasts, in association with epithelial-mesenchymal transition and lymphogenesis, is a critical prognostic parameter in patients with oral tongue squamous cell carcinoma. J Oral Pathol Med 43: 335-343, 2014.

31. Fox SH, Whalen GF, Sanders MM, Burleson JA, Jennings K, Kurtzman S and Kreutzer D: Angiogenesis in normal tissue adjacent to colon cancer. J Surg Oncol 69: 230-234, 1998. 